\title{
Editorial Note: Pediatrics and Health Research - Volume 2, Issue 2
}

\author{
Vihar R Bidwai* \\ Department of Shalya Tantra, Ayurvedic Mahavidyalaya, Pusad, India
}

*Corresponding author: Vihar R. Bidwai, Department of Shalya Tantra, Ayurvedic Mahavidyalaya, Pusad, India, Tel: (07233) 48627; E-mail: vihar4u@gmail.com

Rec date: December 01, 2017; Acc date: December 15, 2017; Pub date: December 27, 2017

Citation: Bidwai VR (2017) Editorial Note: Pediatrics and Health Research - Volume 2, Issue 2. Ped Health Res. Vol. 2 No.3: 21.

\section{Editorial Note}

Children are the future of our world, but unfortunately, they are susceptible to numerous infections and health scares. Child health issues are very much different from those of adults and need special care; therefore, the pediatrics branch of medicine is very important as it specializes in caring for children with health issues. Pediatrics and Health Research is a leading journal in the field of pediatric health. The current issue of Pediatrics and Health Research presents some interesting findings. Kocak et al. [1], compared two commonly used models of growth curves, namely, Gompertz and Logistic models in terms of their accuracy at predicting the height and weight of pediatric patients. Hadeya and Amal [2] assessed the attitude and practices towards oral health, the influence of parental knowledge, and the carie status in Sudanese children suffering from Cerebral Palsy. Hessian et al. [3], analyzed the key determinants of Autism and its prevalence and prediction amongst the population of Dubai. Jayamanne et al. [4], reported a case of a newborn suffering from congenital cytomegalovirus (CMV) infection.

The term growth curve model generally refers to statistical methods which allow for the quantification of inter-individual variability amidst the intra-individual trends over time. Kocak et al. [1], compared two commonly used models of growth curves, namely, Gompertz and Logistic models in terms of their accuracy at predicting the height and weight of pediatric patients. The authors identified that the Gompertz model has a very high standard error of estimation, therefore, should not be recommended for use in clinical trials.

Cerebral palsy (CP) is an umbrella term for referring to a group of permanent disorders which appear early on in childhood. Furthermore, CP patients are at a risk of developing dental caries and other oral diseases. Hadeya and Amal [2] assessed the attitude and practices towards oral health, the influence of parental knowledge, and the carie status in Sudanese children suffering from Cerebral Palsy. The survey revealed that majority of the parents $(90.2 \%)$ thought that good oral health is a crucial factor for optimum general health, $60.2 \%$ of the parents had heard about fluoride, $43.9 \%$ believed that chocolate and soft drinks cause tooth decay, $40.7 \%$ agreed that the media was the main source for information regarding oral health, and $50.4 \%$ of the parents responded that they can achieve good oral health for their children.

Hessian et al. [3], analyzed the key determinants of Autism and its prevalence and prediction amongst the population of Dubai. The results revealed that autism was more prevalent in males ( $84.9 \%$ of the cases) and that the expatriate population was more affected by the condition as opposed to the locals (69.1\% vs $30.9 \%$ ). Additionally, it was also found that $72.4 \%$ of the autism cases were diagnosed as Autism spectrum disorders, 9.2\% as other disorders, and another 9.2\% as ASD features.

The human cytomegalovirus (CMV) is distributed across populations and is recognized as one of the most common causes underlying congenital infection. Due to its wide range of clinical presentations, it poses a diagnostic dilemma for clinicians. Fetal infections are common, as the virus can be transmitted across the placenta; this infection very common in women who suffer from primary infection during pregnancy. Jayamanne et al. [4], reported a case of a newborn suffering from congenital CMV infection, manifesting seizures, recurrent hypoglycaemia, feeding intolerance, and persistent thrombocytopenia.

\section{References}

1. Kocak M (2017) Statistical comparison of growth curve models in first years of life in USA and Turkish Pediatric Populations. Ped Health Res 2: 10.

2. Hadeya MH, Amal HA (2017) Parental oral health knowledge, attitude, practice and caries status of Sudanese cerebral palsy children. Ped Health Res 2: 11.

3. Hessian HY, Abbady KA, Alaam MW (2017) Prevalence, trend, determinants and prediction of autism spectrum disorders among Dubai population: Diagnostic approach and management contexts. Ped Health Res 2: 12.

4. Jayamanne MDCJP, Kumar SV, Kumara SLPC (2017) Feeding intolerance, seizures, recurrent hypoglycemic episodes and persistent thrombocytopenia: A case of symptomatic congenital cytomegalovirs infection. Ped Health Res. 2: 13. 